\title{
Überlegungen zum Verhältnis von Nützlichkeit und Autonomie am Beispiel der Eisenhüttenkunde
}

\author{
VON Stefan KREBS
}

\section{Überblick}

Die Diskussion um die Reform des eisenhüttenkundlichen Studiums, 1902 bis 1906, zeigt anschaulich, wie semantische Bestände aus den Feldern der Politik und der Wirtschaft in das wissenschaftliche Feld transponiert und dort zur Durchsetzung partikularer Interessen eingesetzt wurden. Während der Verhandlungen verwiesen die beiden Aachener Hüttenkundler Fritz Wüst und Wilhelm Borchers auf die besondere Nützlichkeit der Eisenhüttenkunde für die vaterländische Industrie: Nur durch die wissenschaftliche Fundierung der Eisen- und Stahlproduktion könne die deutsche Stahlindustrie im internationalen Wettkampf bestehen. Zugleich erhalte das Kaiserreich damit die notwendigen ,Schutz- und Trutzwaffen', um die deutschen Großmachtambitionen in die Tat umzusetzen, womit der ökonomische Legitimationsdiskurs gleichsam ins politische Feld transzendiert wurde. Der Beitrag untersucht, wie diese Verbindung wissenschaftlicher, ökonomischer und politischer Diskurse semantisch konstruiert und von den Aachener Professoren für den Ausbau ihres Ressourcenensembles instrumentalisiert wurde, dann aber mit den um 1910 einsetzenden Autonomiebestrebungen der Eisenhüttenkunde in Konflikt geriet.

\begin{abstract}
Discussions on reforming the study of ferrous metallurgy in 1902-1906 reveal how semantic contents were transposed from political and economic fields into the scientific field, where they were used to advance particular interests. During the negotiations, the Aachen metallurgists Fritz Wüst and Wilhelm Borchers both pointed to the special utility of ferrous metallurgy for the fatherland's industry: only through a scientific understanding of iron- and steel production could the German steel industry survive in international competition. At the same time, a strong and scientifically-grounded steel industry would provide the Kaiserreich with the arsenal necessary to realize German aspirations to the status of world power, an aspiration which spanned the boundary between discourse on economic legitimation and political discourse. This article investigates the way in which this linkage of scientific, economic, and political discourses was semantically devised, was and how it was used by the Aachen professors to expand their resource ensemble, but also conflicted with ferrous metallurgy's aspirations to autonomy.
\end{abstract}


Am Beispiel der Aachener Eisenhüttenkunde (1870-1914) habe ich in meiner Dissertation gezeigt, wie sich eine technikwissenschaftliche Disziplin im Jahrzehnt vor dem Ersten Weltkrieg durch eine Reihe symbolischer Kämpfe vom Primat ökonomischer Nützlichkeit löste, um nun autonom über die Regeln des Feldes zu bestimmen. ${ }^{1}$ Felder werden hier im Sinne von Pierre Bourdieu als soziale Kräftefelder verstanden, auf denen Akteure gemäß ihrer habituellen Dispositionen und Ressourcen verschiedene Strategien zur Bewahrung oder Verbesserung ihrer sozialen Positionen verfolgen - wobei jedes Feld eine eigene Logik besitzt. Bourdieu hat luzide die Regeln des wissenschaftlichen Feldes beschrieben: Seine Überlegungen lassen sich, ohne dass sie explizit auf die Technikwissenschaften Bezug nehmen, auf diese übertragen. ${ }^{2}$ Neben ökonomischem Kapital spielen auch soziale, kulturelle und symbolische Kapitalien eine entscheidende Rolle bei den Positionierungen im Feld der Wissenschaft. ${ }^{3}$ In den von mir beobachteten Deutungskämpfen zwischen Akteuren aus den eng verflochtenen Feldern von Technikwissenschaft, Wirtschaft und Politik wurden unterschiedliche Vorstellungen zum Verhältnis von Wissenschaft und Wirtschaft deutlich. So galt beispielsweise bis um 1900 in der Hüttenkunde, dass wissenschaftlich wahr sei, was in der betrieblichen Praxis nützlich war. ${ }^{4}$ Im Folgenden werde ich, in Ergänzung zu den Überlegungen in meiner Dissertation, diese Diskurse über die Nützlichkeit der Eisenhüttenkunde genauer untersuchen. Die behauptete Nützlichkeit kann dabei als Teil der symbolischen Ordnung der Technikwis-

1 Stefan Krebs, Technikwissenschaft als soziale Praxis. Über Macht und Autonomie der Aachener Eisenhüttenkunde 1870-1914 (Vierteljahresschrift für Sozial- und Wirtschaftsgeschichte - Beihefte, Bd. 204), Stuttgart 2009. Bei dem vorliegenden Aufsatz handelt es sich um einen überarbeiteten Vortrag, den ich 2009 bei der VDI-Tagung „Flotte, Funk und Fliegen. Leittechnologien der Wilhelminischen Epoche" gehalten habe. Er stellt eine Weiterentwicklung und Zuspitzung meiner Forschungsergebnisse dar.

2 Zum Feld der Wissenschaften vgl. Pierre Bourdieu, Homo academicus, Frankfurt a.M. 1992; ders., Vom Gebrauch der Wissenschaft. Für eine klinische Soziologie des wissenschaftlichen Feldes, Konstanz 1998; ders., Meditationen. Zur Kritik der scholastischen Vernunft, Frankfurt a.M. 2001. Zur interpretativen Offenheit der Theorie der Praxis vgl. Jonathan Sterne, Bourdieu, Technique and Technology, in: Cultural Studies 17, 2003, S. 367-389.

$3 \mathrm{Zu}$ den verschiedenen Kapitalsorten vgl. Pierre Bourdieu, Ökonomisches Kapital, kulturelles Kapital, soziales Kapital, in: Reinhard Kreckel (Hg.), Soziale Ungleichheiten, Göttingen 1983, S. 183-198.

4 Vgl. hierzu den Streit zwischen Fritz Wüst und Adolf Kirdorf um die Zukunft des Thomasverfahrens in Deutschland: Stefan Krebs, „Leben heißt ein Kämpfer sein.“ Zum Verhältnis von Wissenschaft und Öffentlichkeit am Beispiel der modernen Eisenhüttenkunde an der Technischen Hochschule Aachen, in: Berichte zur Wissenschaftsgeschichte 30, 2007, S. 215-229; ders., Die Aachener Thomas-Stahlforschung als kulturelles Kampffeld. Fritz Wüst, der Aachener Hütten-Aktien-Verein und die Forschungen der Technischen Hochschule Aachen zur Verbesserung des Thomas-Verfahrens 1901-1918, in: Manfred Rasch, Jacques Maas u. Manfred Toncourt (Hg.), Das Thomas-Verfahren in Europa. Entstehung, Entwicklung, Ende, Essen 2009, S. 256-271. 
senschaften begriffen werden. ${ }^{5}$ Bei der Beschreibung der Semantik der Nützlichkeit geht es darum, die verschiedenen kulturellen Codes der Nützlichkeit voneinander zu unterscheiden: So stand unter anderem Gemeinwohl gegen wirtschaftlichen oder wissenschaftlichen Eigennutz. Hinzu kam eine symbolische Dimension der Nützlichkeit, die wirtschaftliche Stärke mit dem nationalen Ansehen des Deutschen Kaiserreiches gleichsetzte.

Meine erste These lautet, dass man anhand der Diskussion um den Ausbau der Eisenhüttenkunde nach der Jahrhundertwende exemplarisch die diskursive Aneignung des Wilhelminischen Imperialismus durch die Wissenschaft beobachten kann. Die Aachener Hüttenkundler Wilhelm Borchers ${ }^{6}$ und Fritz Wüst ${ }^{7}$ rekurrierten bei den Verhandlungen zur großen hüttenmännischen Studienreform, die zwischen 1902 und 1906 stattfanden, zunächst auf den gesellschaftlichen Legitimationsdiskurs und verwiesen immer wieder auf die hohe Nützlichkeit der wissenschaftlichen Eisenhüttenkunde für die vaterländische Wirtschaft. Zugleich transzendierten sie diesen ökonomischen Diskurs aber ins politische Feld und versprachen, den Großmachtträumen des Deutschen Kaiserreichs mithilfe der wissenschaftlichen Fundierung der Eisen- und Stahlproduktion die notwendigen Mittel in die Hand zu geben, um diese auch in die Tat umzusetzen.

Der Beitrag untersucht im Folgenden, wie die hier kurz skizzierte Trias von moderner Eisenhüttenkunde, nationalem Wohlstand und deutschen Großmachtinteressen semantisch konstruiert und als diskursive Ressource für den Ausbau der Technikwissenschaften eingesetzt wurde. Dabei geht es um die spezifische Aneignung politisch-ideologischer Diskurse zur Akkumulation von Feldressourcen. Meine zweite These ist, dass die symbiotische Vernetzung von Wissenschaft, Wirtschaft und Politik in eine Krise geraten musste, da die

5 Vgl. dazu die Einleitung in Morten Reitmayer, Elite. Sozialgeschichte einer politischgesellschaftlichen Idee in der frühen Bundesrepublik, München 2009.

6 Wilhelm Borchers (1856-1925) studierte Chemie in Greifswald und Erlangen. Nach seiner Promotion, einigen Jahren praktischer Tätigkeit und einem zweiten Studium der Hüttenkunde an der Bergakademie Clausthal arbeitete Borchers an der Duisburger Hüttenschule, wo er Fritz Wüst kennenlernte. 1897 wechselte er als Dozent für Metallhüttenkunde und Elektrometallurgie an die TH Aachen. Dort setzte er sich u.a. maßgeblich für Wüsts Berufung ein. Paul Röntgen, Wilhelm Borchers, in: Neue Deutsche Biographie, Bd. 2, Berlin 1955, S. 457f.

7 Fritz Wüst (1860-1938) studierte an der TH Stuttgart und der Universität Freiburg, an der er 1886 promovierte. Von 1885 bis 1891 bekleidete er die Stelle eines Chemikers auf dem Königlich Württembergischen Hüttenwerk in Wasseralfingen. Zum 1.4.1891 trat er eine Stelle als Assistent und Lehrer für analytische Chemie an der Maschinenbau- und Hüttenschule in Duisburg an. Seit Januar 1901 vertrat er den erkrankten Friedrich Dürre als Professor für Eisenhüttenkunde an der TH Aachen und wurde zum 1.10.1901 dessen Nachfolger. 1917 wurde er zudem zum Gründungsdirektor des Kaiser-Wilhelm-Instituts für Eisenforschung ernannt. Rheinisch-westfälisches Wirtschaftsarchiv (RWWA), Köln: Abt. 130, 3001030/1, Programm und Jahresbericht der Kgl. Preussischen Maschinenbauund Hüttenschule in Duisburg, Duisburg 1901; Paul Goerens, Friedrich Körber u. Otto Petersen, Nachruf Fritz Wüst, in: Stahl und Eisen 58, 1938, S. 449f. 
Eisenhüttenkunde mit der Jahrhundertwende in ihre Konsolidierungsphase eintrat: Mit der Konsolidierung und der damit einhergehenden Verwissenschaftlichung der Disziplin entwickelte diese eine unabhängige kognitive und soziale Grundlage, die zunächst gegen die politischen und wirtschaftlichen Machtinstanzen erkämpft werden wollte. Das heißt, dass feldexterne politisch-ideologische Ressourcen ihre Akkumulationsfähigkeit innerhalb des wissenschaftlichen Feldes vorübergehend einbüßten: nur um einige Zeit später unter geänderten Vorzeichen einen erneuten Schulterschluss zwischen Wissenschaft, Wirtschaft und Politik zu vollziehen, indem sich Theoriebildung und Grundlagenforschung als neue, nun mittel- und langfristig nützliche Leitideen durchsetzten.

\section{Die Transponierung politisch-ideologischer Diskurse in das Feld der Eisenhüttenkunde}

Am 14. Mai 1902 richtete der Verein Deutscher Eisenhüttenleute (VDEh) eine Eingabe an den preußischen Handelsminister von Möller, deren Wortlaut sogleich in der Vereinszeitschrift Stahl und Eisen veröffentlicht wurde. Ausgangspunkt war die zufriedene Feststellung, ,welch großartigen Aufschwung das Eisenhüttenwesen [d]es Vaterlandes in den letzten Jahrzehnten genommen" habe. ${ }^{8}$ Der Wettlauf mit Großbritannien um die Eisen- und Stahlerzeugung schien noch in den 1880er Jahren aussichtslos, doch durch „rastlose Arbeit" ${ }^{\star 9}$ der deutschen Industrie sei die Stahlerzeugung derart gesteigert worden, dass sie 1894 erstmals über derjenigen der Konkurrentin lag, und auch die Roheisenerzeugung stehe der englischen kaum mehr nach. Zu verdanken habe dies die Stahlindustrie den ,gründlichen wissenschaftlichen Kenntnissen ihrer technischen Kräfte“.${ }^{10}$ Mit Sorge blicke man daher auf den Stillstand des Ausbaus des höheren hüttenmännischen Unterrichtswesens in Preußen: Einzig durch Hebung der hüttenmännischen Bildung an den Technischen Hochschulen und Bergakademien könne Deutschland auch zukünftig den ,scharfe[n] Wettbewerbskampf"11 erfolgreich bestehen.

Der vom VDEh in den Mittelpunkt seiner Eingabe gestellte Wettlauf mit Großbritannien um die höchste Eisen- und Stahlproduktion erscheint im Lichte der Tirpitzschen Flottengesetze und den damit verknüpften Weltmachtträumen des Deutschen Kaisers mehr als ein rein ökonomischer Wettkampf. Die Verbindung von Flottenbau und Stahlproduktion hob vielmehr die Leistungsfähigkeit des Eisenhüttenwesens auf die politische Ebene des Wettkampfs der Nationen empor.

8 O.V., Das höhere hüttenmännische Unterrichtswesen in Preußen, in: Stahl und Eisen 22, 1902, S. 589f., hier S. 589.

9 Ebd.

10 Ebd.

11 Ebd., S. 590. 
Die enge Bindung der Stahlindustrie an die maritimen Großmachtträume Kaiser Wilhelms II. spricht auch aus einem weiteren Bericht in Stahl und Eisen, der einige Wochen nach der obigen Eingabe erschien. Darin drückte der VDEh seine Genugtuung über den Besuch des Kaisers in der Düsseldorfer Industrieausstellung aus. Zentrale Narrative waren die Wettbewerbsfähigkeit der deutschen Industrie gegenüber dem Ausland und die kaiserliche Wertschätzung der Technik: „[K]eine Maschine entging seinem prüfenden Auge und besonders die Vielseitigkeit der Maschinenhalle fand seine rückhaltlose Anerkennung“". ${ }^{12}$ Gegenüber dem Ausstellungsleiter, dem Stahlindustriellen Heinrich Lueg, „,sprach [Wilhelm] seine Ueberzeugung aus, dass die Eisen-, Stahlund Montanindustrie auf dieser Ausstellung jedenfalls dem Auslande gegenüber einen glänzenden Beweis ihrer Wettbewerbsfähigkeit in technischer Beziehung gegeben habe“". ${ }^{13}$ Dieses Lob sei eine große Ermutigung für die rheinisch-westfälischen Industriellen ,noch lange in friedlichem Wettbewerb zum Wohle und zur Ehre des Vaterlandes" ${ }^{\text {"14 }}$ tätig zu sein.

Die Floskel vom friedlichen Wettbewerb war integraler Bestandteil der rhetorischen Rechtfertigung der zunehmend aggressiver werdenden Flottenpolitik: ${ }^{15}$ Die Flotte wurde begriffen als notwendige Schutzmaßnahme für den wirtschaftlichen Interessenstreit mit Großbritannien. Sie war aber auch das umhegte Paradestück deutscher Macht und Größe, und so erregten im Pavillon der Firma Krupp folgerichtig gerade ,[d]ie Panzertürme [...] die besondere Aufmerksamkeit des Kaisers; er stieg in die Thürme und besichtigte ihre Einrichtungen eingehend. Alle Dreh- und Panzerthürme, Strandbatterien und Maschinen wurden in Bewegung oder in Thätigkeit vorgeführt" ${ }^{\text {" }}{ }^{16} \mathrm{Im}$ Rahmen der Ausstellung besiegelte man die Verbundenheit der rheinisch-westfälischen Industrie mit der Kriegsmarine ferner symbolisch durch den mehrwöchigen Aufenthalt des Kanonenboots SMS Panther und des Torpedoboots SMS Sleipner in Düsseldorf. ${ }^{17}$

Die in der Eingabe des VDEh bereits angeklungene Einbindung der Technikwissenschaft Eisenhüttenkunde in den zirkulären Legitimationsdiskurs von wirtschaftlicher Prosperität und imperialer Expansionspolitik, ${ }^{18}$ wurde

12 O.V., Der Kaiser in der Ausstellung, in: Stahl und Eisen 22, 1902, S. 937 ff., hier S. 938

13 Ebd.

14 Ebd., S. 939.

15 Vgl. Manfred Görtemaker, Deutschland im 19. Jahrhundert. Entwicklungslinien, Opladen 1996, S. 362-370.

16 O.V., Der Kaiser in der Ausstellung (wie Anm. 12), S. 938.

17 Mit dem Besuch der beiden Kriegsschiffe sollte u.a. die Flottenbegeisterung der Ausstellungsbesucher entfacht werden, was, wie der Bericht in Stahl und Eisen stolz vermerkte, auch gelang.

18 Die untrennbare Verbindung von Industrie und Flottenpolitik beruhte auf einem selbstreferentiellen Diskurs: Die leistungsstarke Stahl-, Maschinen- und Schiffbauindustrie ermöglichte den großzügigen Flottenausbau, dies steigerte die Umsätze der beteiligten Industriezweige und im nächsten Schritt erforderte das Wirtschaftswachstum koloniale Absatz- und Rohstoffgebiete, deren Sicherung wiederum eine größere Flotte benötigte. 
von den Aachener Professoren bereitwillig aufgegriffen, erhofften sie sich doch Hilfe beim Ausbau ihres Ressourcenensembles. ${ }^{19}$ Zur Verstetigung der Beziehung der Aachener Hochschule zum VDEh initiierte Fritz Wüst die Verleihung der Ehrendoktorwürde an den Vereinsvorsitzenden Karl Lueg und den Geschäftsführer Emil Schrödter. Lueg wurde als ,erfolgreiche[r] Führer im Kampfe um die Unabhängigkeit der vaterländischen metallurgischen Technik, im Streite um den Ruf ihrer Leistungen" ${ }^{20}$ geehrt; Emil Schrödter als „eifrige[r] Vorkämpfer im Streite um den Ruf und die Unabhängigkeit der vaterländischen metallurgischen Technik “. ${ }^{21}$ Die feierliche Übergabe der Doktorurkunden erfolgte am 14. Februar 1903 in Aachen anlässlich der Einweihung des neuen Instituts für Metallhüttenwesen und Elektrometallurgie. In seiner Ehrenpromotion sah Schrödter den Wunsch der Wissenschaft, ,inniger als bisher mit uns Männern der Praxis Hand in Hand zu gehen". ${ }^{22}$ Diese engere Verbindung von Wissenschaft und Wirtschaft sei eine Notwendigkeit der Gegenwart, denn der Eisenhüttenmann könne nur dann seiner Rolle genügen, ,wenn e[r] durch wissenschaftliche Vertiefung für den harten Wettbewerbskampf mit dem Ausland auf die höchste Stufe der Leistungsfähigkeit gebracht wird“. ${ }^{23}$

Die Kooptation der beiden Eisenhüttenmänner in das Feld der Technikwissenschaft, dem sie sich nun enger denn je verbunden fühlten, ${ }^{24}$ und die andauernde Beziehungsarbeit, die Fritz Wüst leistete, um die Kontakte zur rheinisch-westfälischen Stahlindustrie auszubauen und zu pflegen, ${ }^{25}$ trugen rasch dazu bei, zwischen den Aachener Hüttenkundlern und den führenden Männern des VDEh eine Interessenallianz für den Ausbau der Aachener Hochschule zu schmieden. Konkret geschah dies in der Unterrichtskommis-

19 Mitchell G. Ash hat vorgeschlagen, Wissenschaften als Ressourcenensemble zu konzeptionalisieren. Die Verfügung über materielle und immaterielle Ressourcen sind für ihn Voraussetzung dafür, dass einzelne Akteure gestaltend Einfluss auf die Wissenschaftsentwicklung nehmen können, s. ders., Wissenschaften und Politik als Ressourcen für einander, in: Rüdiger vom Bruch u. Brigitte Kaderas (Hg.), Wissenschaften und Wissenschaftspolitik, Stuttgart 2002, S. 32-51; vgl. auch Ulrike Felt, Wissenschaft, Politik und Öffentlichkeit. Wechselwirkungen und Grenzverschiebungen, in: Mitchell G. Ash u. Christian H. Stifter (Hg.), Wissenschaft, Politik und Öffentlichkeit. Von der Wiener Moderne zur Gegenwart, Wien 2002, S. 47-72. Vgl. dazu a. Krebs 2007 (wie Anm. 4).

20 O.V., Das neue elektro-metallurgische Institut in Aachen, in: Stahl und Eisen 23, 1903, S. 297-302, hier S. 297.

21 Ebd.

22 Ebd., S. 298.

23 Ebd.

24 Emil Schrödter hielt, selbst nachdem er sich mit Wüst vollständig überworfen hatte, weiterhin daran fest, dass er der Aachener Hochschule besonderen Dank und Beistand schuldete, vgl. Hauptstaatsarchiv Düsseldorf: Reg. Aachen, Präsidialbüro, Nr. 1301, E. Schrödter an RP Aachen v. 6.8.1909.

25 U.a. fuhr Wüst regelmäßig zum Vereinssitz nach Düsseldorf, um seine Beziehungen zu den Funktionären des VDEh zu pflegen. In einer Briefaktion versuchte Wüst ferner die Alumni des Aachener Instituts als Verbündete zu gewinnen. 
sion des VDEh, der Wüst auf Einladung Schrödters angehörte. ${ }^{26}$ Die in dem Gremium vertretenen Industriellen bereiteten gemeinsam mit Wüst eine zweite Eingabe zur Lage des höheren eisenhüttenmännischen Unterrichtswesens in Preußen vor. ${ }^{27}$ Dabei übernahmen sie dessen Vorschläge bezüglich des konkreten Ausbaus der Aachener Eisenhüttenkunde: Dazu gehörte unter anderem die Forderung nach Gründung einer eigenständigen hüttenkundlichen Fakultät. Diese Forderung war in erster Linie Ausdruck von Wüsts Machtstreben innerhalb der Hochschule. So hoffte er seinen Rivalen, den Vertreter der Anorganischen Chemie Alexander Classen, durch die Fakultätsgründung abdrängen zu können. ${ }^{28}$ Im Gegenzug für die Berücksichtigung seiner akademischen Eigeninteressen zeigte Wüst Verständnis für die sich im Zeichen der Massenstahlherstellung wandelnden Erfordernisse der hüttenmännischen Praxis und plädierte für die Verbesserung der maschinen-technischen Ausbildung innerhalb des hüttenmännischen Studiums. ${ }^{29}$

Die zweite Eingabe, die am 1. August 1903 in Stahl und Eisen veröffentlicht wurde, trug dann deutlich die Handschrift Wüsts. Während zur Begründung knapp auf den Aufschwung der deutschen Stahlindustrie verwiesen wurde, listete die Eingabe detailliert die Forderungen auf, die Wüst der Unterrichtskommission in die Feder diktiert hatte. Als Clou offerierte der VDEh dem Kultusministerium 100.000 Mark für den Neubau eines eisenhüttenkundlichen Instituts in Aachen. Das Geld hatte der Verein auf Betreiben Wüsts aus den erzielten Überschüssen der Düsseldorfer Industrieausstellung erhalten, wobei die Familienbande zwischen Ausstellungsleiter und Vorsitzenden des VDEh hilfreich gewesen sein dürften. Die nun im Namen der Hüttenindustrie geforderte Schaffung einer eigenen Fakultät kaschierte, dass diese

26 Historisches Archiv des Stahlinstitut VDEh (HA VDEh), Düsseldorf: Se 121, E. Schrödter an F. Wüst v. 19.12.1902.

27 O.V., Das höhere eisenhüttenmännische Unterrichtswesen in Preussen, in: Stahl und Eisen 23, 1903, S. 857ff.

28 Die Anorganische Chemie dominierte das eisenhüttenkundliche Curriculum und Classen konnte entsprechend hohe Anteile an den Unterrichtshonoraren für sich verbuchen. Wüsts Studienplanentwurf sah eine starke Reduzierung des anorganischen Unterrichts vor, was von Classen vehement abgelehnt wurde. Die Übungsstunden sollten z.B. von 50 auf 20 herabgesetzt werden. Vgl. Hochschularchiv Aachen (HAAc): 934a, Fritz Wüst, Das Studium des Eisenhüttenwesens und die Errichtung eines neuen eisenhüttenmännischen Instituts an der Königlichen Technischen Hochschule zu Aachen, o.D. (Oktober 1903); auszugsweise erschien die Festschrift auch in Stahl und Eisen: O.V., Zur Ausgestaltung des eisenhüttenmännischen Studiums in Aachen, in: Stahl und Eisen 23, 1903, S. 1257-1268.

29 Die Forderung nach einer besseren maschinen-technischen Ausbildung ergab sich aus den stetig steigenden Anforderungen an die Transport- und Hebevorrichtungen; Fritz Wüst rechnete dem Kultusministerium exemplarisch vor, dass auf einem modernen Hochofenwerk für die Erzeugung von 1.200 Tonnen Roheisen von den Erzen bis zum Kühlwasser täglich rund 33.000 Tonnen bewegt werden müssten. Geheimes Staatsarchiv Preußischer Kulturbesitz, Berlin: I. HA Rep. 76 Kultusministerium, Vb, Sekt. 6, Tit. XV, Nr. 7 Bd. I, Abt. IV, TH Aachen an Kultusministerium v. 20.10.1902. 
Forderung auf die persönlichen Machtambitionen Wüsts zugeschnitten war das heißt, der damit verbundene Eigennutz verschleiert wurde.

\section{Die , wissenschaftliche Fundierung' politisch-ideologischer Diskurse}

Da Fritz Wüst seine Mitarbeit in der Unterrichtskommission nicht offen legte, beauftragte das Kultusministerium ihn als unabhängigen Experten, ein Gutachten zu den Forderungen des VDEh zu schreiben. ${ }^{30}$ Das daraufhin angefertigte Memorandum, das Wüst mit den anderen Mitgliedern der Unterrichtskommission inoffiziell abstimmte, griff die Argumentationslinien der Hüttenindustrie auf und untermauerte sie zugleich durch den Objektivitätsanspruch der Wissenschaft.

Wüst dozierte in seiner Einleitung ausführlich über die Entwicklung der Stahlindustrie in den vergangenen Jahren: ${ }^{31}$ Der Übergang zur Massenstahlherstellung, die Einführung des für Deutschland so wichtigen Thomasprozesses und die Fortschritte der Walzwerktechnik seien nur einige Aspekte „eine[r] vollständigen Umwälzung der Produktionsmethoden“. ${ }^{32}$ Das Eisenhüttenwesen sei ,derart in steter Entwicklung begriffen, dass jede Einrichtung, möge sie noch so vollkommen dem jeweiligen Stand der Eisenhüttentechnik entsprechend gebaut sein, innerhalb 10 Jahren so vollständig überholt ist, dass sie umgebaut werden muss" ${ }^{33}$ Um diesem ständigen betrieblichen Wandlungsprozess zu folgen, fehle der hüttenmännischen Ausbildung die ausreichende Anpassungsfähigkeit. Ein wesentlicher Grund dafür läge darin, dass der preuBische Staat jährlich keine 50.000 Mark für den Spezialunterricht in Eisenhüttenkunde ausgebe. Zum Vergleich führte Wüst die Chemie an, für die mindestens 600.000 Mark aufgewendet würden. Auch die Zahl der etatmäßigen Professuren der beiden Fächer stünde in keinem Verhältnis zu ihrer wahren, wirtschaftlichen Bedeutung, denn für Eisenhüttenkunde gebe es gerade einmal drei Professuren an den Technischen Hochschulen und Bergakademien, für Chemie dagegen allein an den Technischen Hochschulen zwölf und weitere 35 an den Universitäten. Der Wert der von der Eisen- und Stahlindustrie jährlich erzeugten Produkte betrage dagegen drei Milliarden Mark, während derjenige der chemischen Industrie nur 984 Millionen Mark ausmache; der prozentuale Anteil der Löhne an der Wertschöpfung der Eisen- und Stahlindustrie sei zudem besonders hoch, so zahle sie rund eine Milliarde Mark Löhne im Jahr, die chemische Industrie hingegen nur 130 Millionen Mark. Für die Zukunft sei zudem mit ständig wachsender Nachfrage nach Eisen und Stahl zu rechnen. ${ }^{34}$

30 HAAc: 934a, Kultusministerium an Rektor TH Aachen v. 3.9.1903.

31 HAAc: 934a, Fritz Wüst, Das Studium des Eisenhüttenwesens und die Errichtung eines neuen eisenhüttenmännischen Instituts an der Königlichen Technischen Hochschule zu Aachen, o.D. (Oktober 1903).

32 Ebd.

33 Ebd.

34 Ebd. 
Wüsts zahlenmäßige Aufstellung diente im Grunde dazu, die besondere ökonomische Nützlichkeit der Eisenhüttenkunde zu berechnen. Die ganze Beweisführung lief schließlich darauf hinaus, dass der Ausbau der höheren eisenhüttenmännischen Ausbildung nicht im Eigeninteresse der Hüttenkunde und auch weniger in dem der Stahlindustrie als vielmehr im Interesse der vaterländischen Wohlfahrt und damit des Staates selbst begründet liege. Dieser Diskurs der doppelten Uneigennützigkeit gipfelte in der pathetischen Schlussformel: „Deutschland verfügt über reichliche Kohlenlager und über genügend Eisenerze, um noch Jahrhunderte [sic!] hindurch die Stellung unter den eisenerzeugenden Staaten zu behaupten, welche ihm die Intelligenz seiner Ingenieure Schritt für Schritt erobert hat und ihm infolge seiner heutigen Machtstellung zukommt. ${ }^{\text {"35 }}$

\section{Die Technikwissenschaften als ,Schutz- und Trutzwaffen ${ }^{66}$ des deutschen Vaterlandes}

Der öffentlich erzeugte Druck auf die beiden zuständigen Ministerien war so groß, dass sich im Juni 1903 das Handelsministerium dazu entschied, Vertreter aus Wissenschaft, Wirtschaft und Politik zu einer Unterrichtskonferenz nach Berlin einzuladen. ${ }^{37}$

Während der Berliner Verhandlungen bewährte sich die Arbeitsteilung zwischen den Stahlindustriellen und der Aachener Eisenhüttenkunde. Den Grundregeln des wissenschaftlichen Feldes ${ }^{38}$ entsprechend insistierten Wüst und der VDEh bei den Berliner Konferenzen sowie ihren flankierenden Verhandlungen mit dem Kultusministerium, dass sich ihre Forderungen ausschließlich aus den objektiven Bedürfnissen der Praxis ergäben. Die Grundregel, illusio, des wissenschaftlichen Feldes bezeichnet Bourdieu als antiökonomische Ökonomie der Wissenschaft. Das heißt, dass das Interesse der Wissenschaftler im Unterschied zu anderen Feldern nicht als herkömmliches Eigeninteresse auftreten darf. Vielmehr muss das wissenschaftliche Interesse als uneigennützig und unentgeltlich erscheinen. Diese konstitutive Uneigennützigkeit galt es rhetorisch zu verteidigen: Auf die bohrende Nachfrage des Referenten aus dem Kultusministerium, ob die Forderung nach einer eigenen Fakultät nicht nur auf das persönliche Machtstreben Fritz Wüsts zurückgehe, erklärten die Industrievertreter entsprechend eilfertig, dass dies

35 Ebd.

36 Wilhelm Borchers sprach bei der Eröffnungsfeier der TH Breslau 1911 von den Technikwissenschaften als den ,geistigen und substantiellen Schutz- und Trutzwaffen unseres Volkes“, s. o.V., Vereins-Nachrichten. Verein deutscher Eisenhüttenleute, in: Stahl und Eisen 31, 1911, S. 1608-1611 u. 1650f., hier S. 1651.

37 HA VDEh: Se 12 bis a-b, Handelsminister Möller an den VDEh v. 16.6.1903. Die erste Verhandlungsrunde fand am 7.11.1903 statt. Ihr folgte eine zweite Konferenz am 8.1. 1904.

38 Vgl. Krebs (wie Anm. 1), S. 37-46. 
im Gegenteil ihre ureigene Forderung sei. ${ }^{39} \mathrm{Im}$ Gegenzug stützte Wüst mit seiner Autorität als unabhängiger Experte die Forderungen des VDEh..$^{40}$

Zusätzlich semantisch aufgeladen wurde die Diskussion durch die Betonung der ,volkswirtschaftlich höchst wichtige[n] und eminent nationale[n] Bedeutung ${ }^{641}$ des Eisenhüttenwesens, hänge doch von seiner Prosperität maßgeblich die Machtfülle und Weltgeltung des Kaiserreiches ab. Dabei sei einzig die Eisenhüttenkunde als wissenschaftliche Produktivkraft fähig, die Industrie so zu unterstützen, wie es der harte internationale Wettbewerb erzwinge. ${ }^{42}$ Der Verweis, dass das „Ausland uns [in diesem Punkt] vielfach überholt hat" ${ }^{43}$ packte die Kultusbürokratie bei ihrer Ehre: Begriff das ,System Althoff ${ }^{\star}$ doch Wissenschaft als vornehmlich nationalistisches Projekt - als Mittel zur Steigerung des deutschen Ansehens in der Welt. ${ }^{44}$ Die Klagen des Finanzministeriums über die zu veranschlagenden Kosten sowie das ökonomische Interesse der Industrie, konterten die Wirtschaftsvertreter mit einem patriotischen Appell: „Die Opfer müss[t]en nun einmal im Interesse des Vaterlandes gebracht werden" ${ }^{45}$ Schließlich gab Handelsminister Möller den Ausschlag zugunsten der Aachener Hochschule und stellte fest, dass der Studienplan des VDEh die richtige Grundlage dafür bilde. ${ }^{46}$ Dies ist insofern bemerkenswert, als die Technischen Hochschulen nicht länger dem Handelsministerium unterstanden, womit Minister Möller die ihm noch untergeordneten Bergakademien im Stich ließ. Er stellte also die von den Wirtschaftsvertretern diskursiv legitimierte Nützlichkeit der Aachener Eisenhüttenkunde über die Verteidigung seiner Ressortzuständigkeit.

Das heißt, dass den Industriellen das legitime Recht zugebilligt wurde, die Vorgaben für die detaillierte Ausgestaltung des Studiums zu machen. Dies

39 Das Gemeinwohl gehörte als rhetorische Figur seit der Gründung des VDEh zum Repertoire der Eisenhüttenleute. O.V., Technischer Verein für Eisenhüttenwesen II, in: Berggeist 6, 1861, Nr. 22, S. 179.

40 Diese Forderungen waren in weiten Teilen seine eigenen, was die geschickte Rollenverteilung offenbart.

41 HA VDEh: Se 12 bis a-b, Verhandlungen über den Ausbau des Unterrichts im Eisenhüttenwesen an den Technischen Hochschulen und Bergakademien Preußens, Besprechung v. 7.11.1903, Berlin 1903.

42 Die deutsche Eisenhüttenwirtschaft habe zudem mit minderwertigen heimischen Rohstoffen zu kämpfen und dieser Wettbewerbsnachteil sei nur durch die Fortschritte der Eisenhüttenkunde zu kompensieren. Ebd.

43 Ebd.

44 Bernhard vom Brocke, Hochschul- und Wissenschaftspolitik in Preußen und im Deutschen Kaiserreich 1882-1907. Das ,System Althoff“, in: Peter Baumgart (Hg.), Bildungspolitik in Preußen zur Zeit des Kaiserreiches, Stuttgart 1980, S. 9-118, hier S. 15f.

45 HA VDEh: Se 12 bis a-b, Verhandlungen über den Ausbau des Unterrichts im Eisenhüttenwesen an den Technischen Hochschulen und Bergakademien Preußens, Besprechung v. 7.11.1903, Berlin 1903.

46 HA VDEh: Se 12 bis a-b, Verhandlungen über den Ausbau des Unterrichts im Eisenhüttenwesen an den Technischen Hochschulen und Bergakademien Preußens, Besprechung v. 8.1.1904, Berlin 1904. 
verdeutlicht eindrucksvoll die anhaltende Macht der hüttenmännischen Praxis über die Eisenhüttenkunde - ein typisches Merkmal der technikwissenschaftlichen Disziplingenese während der Herausbildungsphase ${ }^{47} \mathrm{Zu}$ bedenken ist allerdings, dass es gerade Fritz Wüst als Wissenschaftler war, der zur Kaschierung seiner Eigeninteressen diesen Übergriff der Praxis inszenierte und zu seinem Vorteil zu nutzen verstand: ${ }^{48}$ Konnte er doch mithilfe des VDEh den wissenschaftlichen Wettbewerb im Feld der Eisenhüttenkunde suspendieren und den einseitigen Ausbau seines Ressourcenensembles durchsetzen. ${ }^{49}$ Ein lohnendes Arrangement für die Aachener Eisenhüttenkunde, die nun das Privileg genoss, als erster Standort den neuen Reformstudiengang einzuführen, hierfür zusätzliche Professuren und Dozenturen und schließlich den 1,5 Millionen Mark teuren Neubau eines Lehr- und Forschungsinstituts für die gesamte Hüttenkunde erhielt. Durch den explosionsartigen institutionellen Ausbau und den damit verbundenen Prestigegewinn entwickelte der Aachener Studiengang eine ungeheure Anziehungskraft, so dass sein Anteil an den Einschreibungen für Hüttenkunde bis auf über 50\% emporschnellte. ${ }^{50}$

An der „großen hüttenmännischen Reform“51 lässt sich anschaulich die Akkumulationsfähigkeit exoterer Diskurse im Feld der Wissenschaft aufzeigen: Nicht der wissenschaftliche Wandel begründete die Umgestaltung des Studiums, sondern die Veränderungen der industriellen Praxis machten diesen zur Notwendigkeit. Allerdings reichte dies allein nicht aus: Erst der Rekurs auf die Verbindung von wirtschaftlicher Prosperität und deutscher Weltgeltung gab den Ausschlag zugunsten des Ressourcenausbaus der Eisenhüttenkunde. Das heißt, der Gebrauch der diskursiven Codes des Imperialismus verschaffte den Aachener Eisenhüttenkundlern einen Legitimationsvorsprung. Mit Morten Reitmayer kann man sagen, dass der Gebrauch der imperialen Rhetorik als Teil der sprachlichen Codes der technikwissen-

47 Die Periodisierung geht zurück auf das Dresdener Konzept zur Genese technikwissenschaftlicher Disziplinen. Hier und im Folgenden vgl. Martin Guntau u. Hubert Laitko (Hg.), Der Ursprung der modernen Wissenschaften. Studien zur Entstehung wissenschaftlicher Disziplinen, Berlin 1987; Thomas Hänseroth u. Klaus Mauersberger, Das Dresdener Konzept zur Genese technikwissenschaftlicher Disziplinen, eine Bilanz, in: Dresdener Beiträge zur Geschichte der Technikwissenschaften 24, 1996, S. 20-45.

48 Die Inszenierung bestand u.a. darin, dass sich die Aachener Professoren gemeinsam mit den Vertretern des VDEh absprachen und gezielt die unterschiedlichen Rollen für die bevorstehenden Konferenzen verteilten: So trug beispielsweise Wüst die Klagen des VDEh vor, während Fritz Kintzlé und Friedrich Springorum den gewünschten Zuschnitt des neuen Curriculums erläuterten. Vgl. Krebs (wie Anm. 1), S. 236-269.

49 Zur Suspendierung des Wettbewerbs in wissenschaftlichen Feldern vgl. Bourdieu 1998 (wie Anm. 2), S. 28.

50 Während des Ersten Weltkrieges stieg er bis auf 59\% an, der größte Verlierer war die Berliner Bergakademie, deren Studierendenzahl komplett zusammenbrach, so dass sie schließlich 1916 mit der Technischen Hochschule Berlin fusionieren musste. Vgl. die statistischen Angaben in Stahl und Eisen 1906-1914.

51 HA VDEh: Se 12bis, Fachabteilung für Hüttenkunde an der Technischen Hochschule Aachen, Zur Ausbildung der Hütteningenieure, Düsseldorf 1925. 
schaftlichen Nützlichkeit, das politisch-ideelle Potenzial der Nützlichkeitssemantik aktivierte und die Akteure des politischen Feldes zum Handeln zugunsten der Aachener Hochschule zwang. ${ }^{52}$ Es lässt sich festhalten, dass in der Studienreformdebatte unterschiedliche Interessenskomplexe zur Deckung kamen. Im Fall der nationalen Machtpolitik konnten Wirtschaft und Wissenschaft geltend machen, dass sie gemeinsam zu Ruhm und Größe des Deutschen Kaiserreiches beitrugen - ein Ziel, das vom Kultusministerium geteilt und deshalb unterstützt wurde. Zentrale Legitimationsfigur war die postulierte Uneigennützigkeit der jeweiligen Forderungen, wobei sich die unterschiedlichen Akteure gegenseitig in ihren Selbstdeutungen bestärkten. Besonders sichtbar wird dies anhand der Verhandlungstaktik des VDEh und der Aachener Eisenhüttenkunde, die mit ihren verteilten Rollen virtuos die Forderungen der anderen Seite als legitim anerkannte oder gar als die eigenen ausgab. Mit Pierre Bourdieu kann hier von einem Kreislauf wechselseitiger Legitimation gesprochen werden - einem Kreislauf, in dem die Akteure voneinander getrennter Felder symbolische Dienste füreinander leisteten.$^{53}$

Die Einheit der nationalen Trias aus Stahlindustrie, Eisenhüttenkunde und Großmachtpolitik wurde anlässlich der Grundsteinlegung für das neue hüttenkundliche Institut am 16. Juni 1906 noch einmal feierlich beschworen. ${ }^{54}$ Der Vorsitzende des VDEh Friedrich Springorum freute sich darüber, dass die von der Praxis aufgestellten Grundsätze als richtig anerkannt worden seien. Wilhelm Borchers begrüßte als Rektor emphatisch die Nützlichkeitserwartungen, die an die Hüttenkunde von Seiten der Wirtschaft und der Politik gestellt wurden. Fritz Wüst beendete seine Ansprache mit dem Schwur, dass die zukünftigen Absolventen ,dem Deutschthum [nicht] verloren gehen [und] das größte Ideal verwirklichen [werden], welches sich ein deutschfühlender Mann jemals setzen kann, d.h., Sie werden zum Ruhme und zur Größe unseres geliebten Vaterlandes das Ihrige beitragen" ${ }^{55}$ Entsprechend dieser national-chauvinistischen Rhetorik erklangen auch die Sinnsprüche zur eigentlichen Grundsteinlegung. Wüst proklamierte für die Eisenhüttenkunde: „Möge die Tätigkeit in diesem Institut der deutschen Hüttenindustrie zu Nutz und Frommen gereichen.“ Adolf Kirdorf rief im Namen der Stahlindustrie aus: „Wer die Jugend hat, hat die Zukunft." Und Regierungsrat Robert von Goerschen fasste seine Erwartungen an das neue Institut zusammen: „Zu Deutschlands Größe beim Wettbewerb der Völker. ${ }^{\text {"56 }}$ Das diskursive Band zwischen Eisenhüttenkunde, Stahlindustrie und dem Deutschen Kaiserreich schien damit besiegelt.

52 Reitmayer (wie Anm. 5), S. 36.

$53 \mathrm{Zu}$ den Legitimationskreisläufen in ausdifferenzierten Gesellschaften vgl. Bourdieu 2001 (wie Anm. 2), S. 130-135.

54 O.V., Institut für das gesamte Hüttenwesen in Aachen, in: Stahl und Eisen 26, 1906, S. 806-809.

55 Ebd., S. 809.

56 Alle Aussprüche ebd. 
Die gezeigte rhetorische Aneignung des Wilhelminischen Imperialismus durch die Aachener Eisenhüttenkunde muss im Kontext des Nützlichkeitsdiskurses der Technikwissenschaften der Zeit gesehen werden. So zeigte bereits der Wettstreit zwischen Danzig und Breslau um den Standort für die Neugründung einer Technischen Hochschule in den 1890er Jahren, wie beide Seiten auf ähnliche rhetorisch-diskursive Strategien zurückgriffen, um sich selbst ins rechte Licht zu rücken. ${ }^{57}$ Die Vertreter der Stadt Breslau verwiesen auf die ökonomische Nützlichkeit einer Technischen Hochschule für das oberschlesische Industriegebiet. ${ }^{58}$ Dagegen setzte Gustav von Goßler als amtierender Oberpräsident der Provinz Westpreußen ein politisch-ideologisches Projekt, das schließlich den Ausschlag zugunsten Danzigs geben sollte. Er stilisierte Danzig in seiner Denkschrift betreffend der Gründung einer Technischen Hochschule zum „Hort des Deutschthums“ im Osten. Die neue Lehranstalt solle das Selbstbewusstsein der Provinz stärken und den Zuzug deutscher Arbeiter und Ingenieure fördern. Ferner avisierte Goßler als mögliche fachliche Schwerpunkte den Schiffbau und Schiffsmaschinenbau. Insgesamt, so Wolfgang König, las sich Goßlers Denkschrift ,,wie eine Aufzählung der bekannten kaiserlichen Interessen" ${ }^{59}$ Hier könnte man ergänzen, dass sich Goßlers Denkschrift zugleich wie eine Aufzählung der Nützlichkeitscodes der Zeit liest.

\section{Die Autonomisierung der Eisenhüttenkunde als Abkehr vom Nützlichkeitskalkül?}

Die erfolgreiche Aneignung der politisch-ideologischen Semantik des deutschen Imperialismus leitete eine ungeheure Ressourcenakkumulation der Aachener Eisenhüttenkunde ein. Anstatt aber die vom VDEh erhobenen Nützlichkeitserwartungen zu erfüllen, setzte Fritz Wüst in den folgenden Jahren erfolgreich sein symbolisches Kapital ein, um die Definitionsmacht über die Regeln des eisenhüttenkundlichen Feldes zu erlangen. Damit katapultierte er die gesamte Disziplin in ihre Konsolidierungsphase und veränderte in letzter Konsequenz das gesamte Feld der Eisenhüttenkunde. Neben der Hinwendung zu neuen Erkenntnisgegenständen führte vor allem die Adaption neuer Forschungsmethoden und die Ausrichtung der wissenschaftlichen Tätigkeit auf die Theoriebildung dazu, dass sich die Regeln des Feldes wandelten. ${ }^{60}$

57 Die Zunahme der Studentenzahlen in der 1890er Jahren war der willkommene Anlass, die seit den 1860er Jahren diskutierte Gründung einer Technischen Hochschule im Nordosten Preußens wiederzubeleben. Während auf die sechs Hochschulen in den übrigen deutschen Staaten rund 6.500 Studierende kamen, verzeichneten die drei preußischen Hochschulen allein einen Anstieg auf rund 5.200 Studierende. O.V., Technische Hochschule in Danzig, in: Stahl und Eisen 19, 1899, S. 301f.

58 Wolfgang König, Wilhelm II. und die Moderne. Der Kaiser und die technisch-industrielle Welt, Paderborn u.a. 2007, S. 127.

59 Ebd.

60 Die deutsche Eisenhüttenkunde suchte und fand den Anschluss an den Forschungsstand in Frankreich, Großbritannien und den Vereinigten Staaten. Hierdurch und die Adaption 
Dazu gehörte, dass sich die Eisenhüttenkunde gegen den Widerstand des VDEh das Recht erkämpfte, als Wissenschaft autonom darüber entscheiden zu können, was wissenschaftlich wahr und nützlich ist. Damit stand die Leitidee der Herausbildungsphase zur Disposition, dass wissenschaftlich wahr ist, was der Industrie ökonomischen Nutzen bringt. ${ }^{61}$

Zielten die geänderten Erkenntnis- und Produktionsmodi im Kern auf die Verwissenschaftlichung der Eisenhüttenkunde, führten die damit einhergehenden Veränderungen des eisenhüttenkundlichen Feldes dazu, dass sich auf ihm neue Positionen und damit neue Regeln für den Erwerb wissenschaftlichen Kapitals etablierten. Als neue Leitwährung setzten sich Originalbeiträge durch, die sich vornehmlich der eisenhüttenkundlichen Grundlagenforschung widmeten - voran die Präzisierung und Interpretation des Eisen-Kohlenstoff-Diagramms. Rund die Hälfte der zwischen 1903 und 1914 entstandenen Doktorarbeiten wandte sich allein diesem neuen Forschungsgebiet zu. Sichtbar wird die neue Trennung von Wissenschaft und Wirtschaft in der konfliktgeladenen Ausdifferenzierung des eisenhüttenkundlichen Zeitschriftenwesens. Der VDEh versuchte zunächst das Publikationsmonopol der Vereinszeitschrift Stahl und Eisen zu bewahren. Die Vereinsvertreter mussten aber schließlich, trotz ihres anfänglichen ökonomischen Boykotts, das Existenzrecht einer Spezialzeitschrift, der Metallurgie, anerkennen. Diese von Wüst und Borchers begründete Zeitschrift wandte sich ganz der theoretischen Seite der Hüttenkunde zu. Damit suspendierte die Eisenhüttenkunde vorübergehend die Besonderheit der Technikwissenschaften, nämlich dass sie zwei Abnehmerkreise für ihre Forschungsergebnisse hat: die anderen Technikwissenschaftler und die Ingenieure in der technisch-industriellen Praxis. Letztere partizipierten aber nicht an den neuen Erkenntnissen, so dass sich der Abnehmerkreis entsprechend reduzierte. Die Eisenhüttenkundler konnten ihrerseits unter den neuen konsolidierten Produktionsbedingungen, den Regeln des wissenschaftlichen Feldes entsprechend, nur von den anderen Mitgliedern der Scientific Community im engeren Sinne Anerkennung für ihre Forschungsleistungen erhalten. ${ }^{62}$ Der Verweis auf die etwaige Nützlichkeit der Forschungsergebnisse in der betriebli-

neuer Methoden und Theorieansätze, die von benachbarten Disziplinen wie der Physikalischen Chemie bereits entwickelt worden waren, konnte die Eisenhüttenkunde sehr schnell ein auf die Verwissenschaftlichung der eigenen Disziplin abzielendes Forschungsprogramm initiieren. Vgl. Nicole Chezeau, De la Forge au Laboratoire. Naissance de la métallurgie physique 1860-1914, Rennes 2004.

61 Vgl. zum Streit um das Primat der Ökonomie im eisenhüttenkundlichen Feld die Auseinandersetzungen zwischen Wüsts Vorgänger Ernst Friedrich Dürre und dem Direktor der Friedrich-Wilhelmshütte Joseph Schlink (1877-1879) sowie zwischen Fritz Wüst und Adolf Kirdorf (1909). Krebs (wie Anm. 1), S. 99-115; ders. 2007 (wie Anm. 4), S. 222-225.

62 Im Sinne Ludwik Flecks verschob sich mit der Konsolidierung die Grenze zwischen dem esoterischen und dem exoterischen Kreis der Eisenhüttenkunde, vgl. ders., Entstehung und Entwicklung einer wissenschaftlichen Tatsache. Einführung in die Lehre vom Denkstil und Denkkollektiv, Frankfurt a.M. 1980. 
chen Praxis war kein ausreichendes Kriterium mehr, um wissenschaftliches Kapital zu erwerben. Dies bedeutete mithin eine starke Abgrenzung vom ökonomischen Feld des Eisenhüttenwesens und negierte das zuvor gehuldigte Nützlichkeitskalkül - waren die Ergebnisse der Grundlagenforschung doch scheinbar nicht in der betrieblichen Praxis verwertbar. ${ }^{63}$

Zur gleichen Zeit, als der Bruch zwischen Eisenhüttenkunde und der hüttenmännischen Praxis zementiert schien, bildete sich mit der 1911 erfolgten Gründung der Kaiser-Wilhelm-Gesellschaft schrittweise das Paradigma von der wirtschaftlichen Nützlichkeit der Grundlagenforschung heraus. 1917 schwenkte auch der VDEh auf die neue Linie ein, indem er sich für die Errichtung eines Kaiser-Wilhelm-Instituts für Eisenforschung einsetzte: „Es soll vorausschauend der Technik den Weg weisen und die Grundlagen schaffen, die nötig sind, um unser Eisenhüttenwesen dauernd mit an der Spitze zu halten. ${ }^{\prime 64}$ Trotz der erfolgten Umkehr der Vorzeichen ${ }^{65}$ erscheint die semantische Zurichtung der eisenhüttenkundlichen Grundlagenforschung als gewinnbringend für die Praxis wie eine Rückkehr zum Status quo ante. Der veränderte Erwartungshorizont, also die Erwartung, dass sich die Grundlagenforschung nur mittel- und langfristig als nützlich erweisen sollte, ermöglichte den Akteuren ein größeres Maß an Autonomie, entband sie aber nicht grundsätzlich von den an sie gestellten Nützlichkeitserwartungen. Diese lassen sich auch daran bemessen, dass die im VDEh organisierten Werke bereit waren, Gelder in bislang ungekannter Höhe in den Aufbau des neuen Forschungsinstituts zu investieren. Auf Seiten der Politik bestand dagegen, wie schon in den Berliner Verhandlungen 1904 bis 1906, die Befürchtung, dass das zu gründende Institut der rheinisch-westfälischen Stahlindustrie und deren Eigeninteressen zu nahe stehen könnte: die Autonomie der Institution und damit die gesamtgesellschaftliche Nützlichkeit also in Frage stehe ${ }^{66}$ Insofern erscheint es folgerichtig, dass ausgerechnet Fritz Wüst, der zuvor die Trennung der Eisenhüttenkunde von der Stahlindustrie maßgeblich betrieben und sich so einen Ruf als unabhängiger Wissenschaftler erworben hatte, zum Gründungsdirektor bestellt wurde: Die eisenhüttenkundliche Grundlagenforschung war damit als Mittel zum Zweck, der weiterhin Ruhm und

63 Die mangelnde Übertragbarkeit und Anwendbarkeit der neuen Erkenntnisse und Methoden lag u.a. in der mangelhaften Praxistauglichkeit der neuen Werkzeuge und Instrumente; besonders der Schnellbetrieb des Thomasprozesses sperrte sich gegen die Einführung neuer Analysemethoden.

64 RWWA: Abt. 130, 300126/25, Niederschrift der Sitzung des VDEh am 19.6.1917.

65 Das Ziel der Technikwissenschaften war nun nicht länger die nachgelagerte Ordnung und Systematisierung des Erfahrungswissens, sondern die voranschreitende Experimentalforschung und Theoriebildung.

66 Archiv zur Geschichte der Max-Planck-Gesellschaft, Berlin: I. Abt., Rep. 16, Nr. 1-1, F. Wüst an O. Petersen v. 20.4.1917; F. Wüst an A. Vögler v. 20.4.1917; F. Schmidt an F. Wüst v. 28.4.1917. 
Größe des Deutschen Vaterlandes hieß, anerkannt - und ein prekärer Ausgleich zwischen Nützlichkeit und Autonomie gefunden.

Anschrift des Verfassers: Dr. Stefan Krebs, Department of Technology, Innovation \& Society, Eindhoven University of Technology, IPO 1.16, P.O. Box 513, 5600 MB Eindhoven, Niederlande, E-Mail: s.krebs@tue.nl 